\title{
Molecular Dynamics Study of the Dynamics Near the Glass Transition in Ionic Liquids
}

\author{
J. HABASAKI ${ }^{* \dagger}$ and K. L. NGaI** \\ *Tokyo Institute of Technology, 4259 Nagatsuta, Yokohama 226-8502, Japan \\ **Naval Research Laboratory, Washington, D.C. 20375-5320, USA
}

\begin{abstract}
Molecular dynamics (MD) simulations have been performed to study the dynamics near the glass transition regime of molecular ions in ionic liquids. The glass transition temperature in the simulated 1-ethyl-3-methyl imidazolium nitrate $\left(\mathrm{EMIM}-\mathrm{NO}_{3}\right.$ ) system was determined by plotting density against temperatures. The dynamics at several temperatures in the liquid, supercooled liquid, and glassy states have been characterized by the diffusion coefficients, fractal dimension analysis of the trajectories, and the van-Hove functions. The diffusion coefficient approximately obeys the Vogel-FulcherTammann (VFT) relation. However, two power laws or two exponentials are also good descriptions of the data. The fractal dimension of the random walks is a measure of the complexity of the trajectory, which is attributed to the geometrical correlations among successive motions. Rapid increase of the fractal dimension of the random walks on decreasing temperature is found for both cations and anions. Temperature dependence of the fractal dimension of the random walks for the long range (accelerated) motion is larger than that for short range (localized) motion. This reasonably explains the change in the slopes found in the temperature dependence of the diffusion coefficients. At around the glass transition temperature, long range motion is essentially absent during the observed times, up to several nano seconds. This feature is also confirmed by the van-Hove functions. Such slowing down of the dynamics in the fragile ionic liquids is characterized by the changes from long range motion to short range motion instead of sudden changes at around $T_{0}$ in the VFT relation.
\end{abstract}

(Received March 31, 2008; Accepted June 11, 2008; Published October 10, 2008)

\section{Introduction}

A new class of chemical compounds related to molten salts is now called the room-temperature ionic liquids (ILs), and it has attracted much attention in the research community because they possess many desirable properties. Typical ILs of interest are salts each of which is constituted by an organic cation and an inorganic anion, and their melting points are below or near the room temperature. ${ }^{1-4}$ The properties include high thermal stability, negligible vapor pressure and good solvents for many substances with negligible vapor pressure, making them replacements for volatile organic solvents in various applications. The ILs also have high ionic conductivity values suitable for use as electrolytes.

Although ILs are liquids over wide temperature ranges, they can be vitrified at sufficiently low temperatures or elevated pressures. In the present work, our attention is focused on the changes of dynamics near the glass transition. The purpose of this paper is to explain what kind of microscopic dynamics is responsible for the changes in the dynamics near the glass transition. The dynamics at several temperatures in the liquid, supercooled liquid, and glassy states have been characterized by van-Hove functions and also by the fractal dimension analysis of the trajectories. Since the dynamics of ILs show several properties common to those of other glass forming materials, we

† To whom correspondence should be addressed.

E-mail: habasaki.j.aa@m.titech.ac.jp can expect that the glass transitions in other systems can be characterized in a similar manner.

Molecular dynamics (MD) simulations are useful methods to learn more about the mechanism of the transport properties as well as the mechanism of the glass transition macroscopically and microscopically. In MD simulations, time developments of positions of atoms in the system are calculated using the equation of the motion of atoms that takes into account the many body interactions. The system has the general character of a model system to study the glass transition, and at the same time it is realistic enough to compare with experimental findings. Study of the microscopic mechanism of the slowing down of the dynamics in ILs by MD simulations will be useful for the application of ILs in many analytical sciences as well as for the developments in glass physics and chemistry.

\section{Molecular Dynamics Simulations}

We study the dynamics of the IL, 1-ethyl-3-methyl-imidazolium nitrate $\left(\mathrm{EMIM}-\mathrm{NO}_{3}\right)$ by using the potential functions of the following form;

$$
\begin{aligned}
U(R)= & \sum_{\text {bonds }} K_{\mathrm{r}}\left(r-r_{\mathrm{eq}}\right)^{2}+ \\
& \sum_{\text {angles }} K_{\theta}\left(\theta-\theta_{\mathrm{eq}}\right)^{2}+ \\
& \sum_{\text {dihedrals }} \frac{V_{\mathrm{n}}}{2}(1+\cos [n \phi-\gamma])+
\end{aligned}
$$




$$
\begin{aligned}
& \sum_{\mathrm{i}<\mathrm{j}}^{\text {atoms }}\left(\frac{A_{\mathrm{ij}}}{R_{\mathrm{ij}}^{12}}-\frac{B_{\mathrm{ij}}}{R_{\mathrm{ij}}^{6}}\right)+ \\
& \sum_{\mathrm{i}<\mathrm{j}}^{\text {atoms }} \frac{q_{\mathrm{i}} q_{\mathrm{j}}}{\varepsilon R_{\mathrm{ij}}}
\end{aligned}
$$

similar to those used in previous works on ILs. ${ }^{5-10}$

The model uses a sum of bond, angle, and dihedral deformation energies, a pairwise standard $(6,12)$ Lennard-Jones potential representing van der Waals interactions, and Coulombic interactions between atoms with charges $q_{\mathrm{i}}$. The parameters therein were taken from the General Amber Force Field. ${ }^{11}$

The equilibrated density was 1.206 at $400 \mathrm{~K}$. The system in the present work can be regarded as a useful model for considerations of glass transitions of ILs.

MD simulations were performed using this force field on smaller systems having $64 \mathrm{EMIM}^{+}$and $64 \mathrm{NO}_{3}{ }^{-}$, and on a larger system having $256 \mathrm{EMIM}^{+}$and $256 \mathrm{NO}_{3}{ }^{-}$with a total of 5888 ions. We found that the system size dependence of the diffusivity of the system is small; hence, we use results of both systems for discussion. Periodic boundary conditions were imposed, and Coulomb interactions were calculated using the particle mesh Ewald method. The system was equilibrated at $3000 \mathrm{~K}$ and the temperature was gradually decreased. The time step was either 1 or $2 \mathrm{fs}$, and the simulations of NVE ensemble were carried out up to 2.5 or $10 \mathrm{~ns}$ at temperatures equal to 0 $250,300,350,370,400,500,600,800$ and $1000 \mathrm{~K}$ after equilibration with a more than $1 \mathrm{~ns}$ run of NPT ensemble at each temperature. Some additional data points are used in several figures to be presented later.

We examine the fractal dimension of the random walk of the trajectories, $d_{\mathrm{w}}$, by the divider method, ${ }^{12}$ which was found to be useful to analyze the trajectory of ions in other ionic systems. ${ }^{13}$ When the length of a complicated trajectory obtained by simulation during $t_{\mathrm{run}}$ is measured by a divider (i.e., a straight ruler) of length $L$, the number of times needed for the divider to cover the entire trajectory is $v$. The total length of the trajectory is $v L$, which depends on the choice of $L$. For more complicated trajectories, a larger $v L$ is obtained if a smaller $L$ is used. For any $L$, the sum of $v$ for all trajectories is $N$. Then, $N$ is the number of times required for the divider of length $L$ to cover all the trajectories obtained after a run up to $t_{\text {run }}$. In this manner, we can examine all the trajectories of the ions obtained during runs at several temperatures, with $L$ ranging from 0.3 to $10 \AA$. $N$ is determined as a function of $L$. From the slope in the double logarithm plots we determine the fractal dimension of random walk, $d_{\mathrm{w}}$, defined by

$$
N=A L^{-\mathrm{d}_{w}} \text {. }
$$

\section{Results and Discussion}

Experimentally, the melting point of the system is known to be $313 \mathrm{~K}$ and crystallization occurs at about $290 \mathrm{~K} .{ }^{14}$ Although the system is known to show crystallization, by rapidly quenching in the simulation a change of the slope is found in the plot of density versus temperature (Fig. 1). The glass transition temperature determined in the manner is shown in Fig. 1, where the density was obtained by NPT ensemble simulations. The glass transition of the simulated system is determined to be 260 $\mathrm{K}$, which is about 50 degrees higher than the value estimated from the experimental melting point based on the $2 / 3$ rule. $\left[T_{\mathrm{g}} / T_{\mathrm{m}}=2 / 3\right]$. The rule holds well in the ionic liquid 1-butyl-

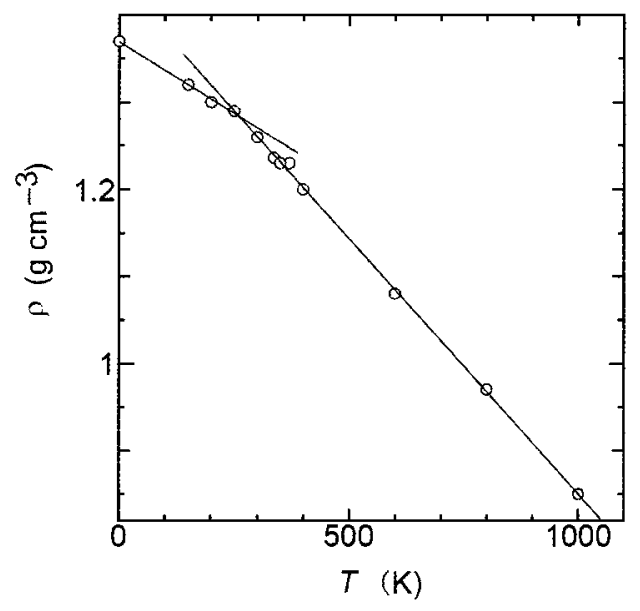

Fig. 1 Density against temperature plot of EMIM-NO${ }_{3}$ obtained by the MD simulation. The glass transition temperature obtained from the change in temperature dependence of the density of the simulated system is $260 \mathrm{~K}$.

3-methyl imidazolium chloride..$^{15}$

Thus, at temperatures less than $260 \mathrm{~K}$, the system is in the glassy state. Between $260 \mathrm{~K}$ and about $360-370 \mathrm{~K}$ in the simulation, the system can be regarded as being in the supercooled liquid state. The glass transition temperature so determined is useful for comparison of the simulated system with experimental data of the real system. However, the $T_{\mathrm{g}}$ determined by the density-temperature plot is not necessarily equal to the one defined by dynamics. Dynamical slowing down similar to the present work has been found in other systems such as binary Lennard-Jones systems. ${ }^{16,17}$ The slowing down is observed even in the fixed volume conditions; this means that the change in the density dependence is not uniquely connected to the dynamical slowing down. Slowing down of the dynamics is characterized by the change of the diffusion coefficients. Diffusivity above $800 \mathrm{~K}$ seems to show the Arrhenius type temperature dependence. Below this temperature the system shows the dynamical slowing down by following the VogelFulcher-Tammann (VFT) dependence;

$$
D=D_{0} \exp \left(\frac{E}{T-T_{0}}\right) \text {. }
$$

The VFT fit to the MD simulation data is shown by the curve in Fig. 2. Behavior of the anions is similar to that of cations. In several ionic liquids so far, diffusivity, as well as conductivity can be well fitted ${ }^{18,19}$ to the VFT equation. ${ }^{20}$ For cations and anions, $T_{0}$ obtained from the fits are 243.2 and $256.5 \mathrm{~K}$, respectively.

The value of $T_{0}$ in Eq. (3), at which the diffusion coefficient is practically 0 , is slightly lower than the glass transition temperature obtained from the density-temperature plot. The relation $T_{0}<T_{\mathrm{g}}$ is also known for the viscosity $\eta$, which was also fitted to $\eta \propto \exp \left[D T_{0} /\left(T-T_{0}\right)\right] .{ }^{20}$ The results in the present work are consistent with the analysis in previous works using VFT type equation; however, the $T_{0}$ value is not necessarily a true characteristic temperature of the system for the following reasons. Although the VFT relation approximately holds, data for both high and low temperature region can also be well fitted by exponentials, as shown in Fig. 2(b). Fitting by power laws is also possible. The VFT fit is not necessarily the best fit in both in high and low temperature regions. At least we can say that the existence of $T_{0}$ is due to a choice of the functional form and 


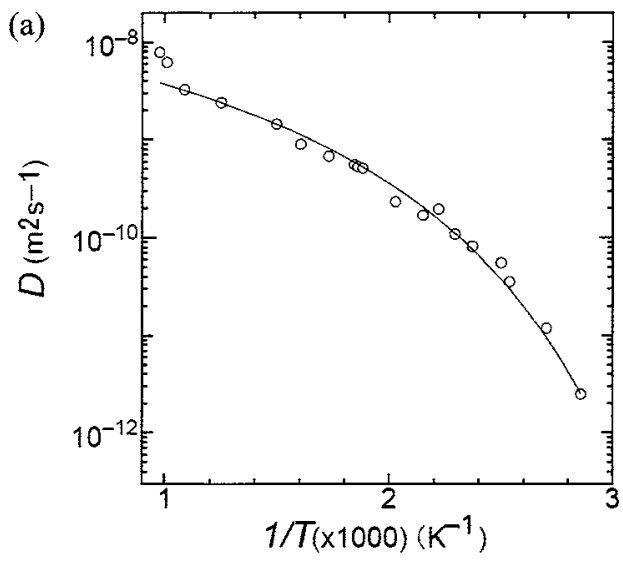

(b)

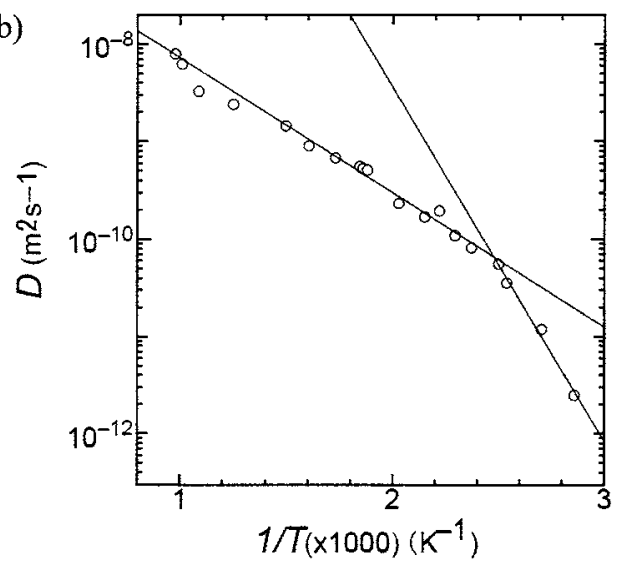

Fig. 2 (a) VFT fit of the diffusion coefficient of cations. (b) Exponential fit of the diffusion coefficient of cations. The same data are used in both (a) and (b).

is not necessarily a true characteristic temperature of the system. In fits using exponential functions and in those using power law functions, we have observed an inflection point at around $410 \mathrm{~K}$. From the observation of the inflection, the rapid slowing down of the dynamics at lower temperature region is attributed to the coexistence of a gentle slope at higher temperature and a steeper slope at lower temperature regimes rather than to the characteristics near the specific temperature $T_{0}$. Due to the limited range of each temperature region, it is difficult to judge which expression is the best by the quality of the fitting only. In the following section, we will show that the inflections of the dynamics correspond to changes in the characteristics in trajectories of ions.

If the functional form at lower temperature region is exponential, the extrapolated value of the diffusion coefficient of cation becomes $8.6 \times 10^{-23} \mathrm{~m}^{2} \mathrm{~s}^{-1}$ at $200 \mathrm{~K}$ and $3.8 \times 10^{-40} \mathrm{~m}^{2}$ $\mathrm{s}^{-1}$ at $100 \mathrm{~K}$. It must be difficult to observe the diffusion in the MD simulation up to several nano seconds as well as by most of experimental methods at ordinary time scales. Both in this case and in the power law case, the use of a phase transition to explain the glass transition is not necessary, although the results do not exclude the possibility of the existence of other characteristic temperatures.

\section{Fractal Dimension of the Random Walks}

In general, dynamics of ions in disordered materials show large

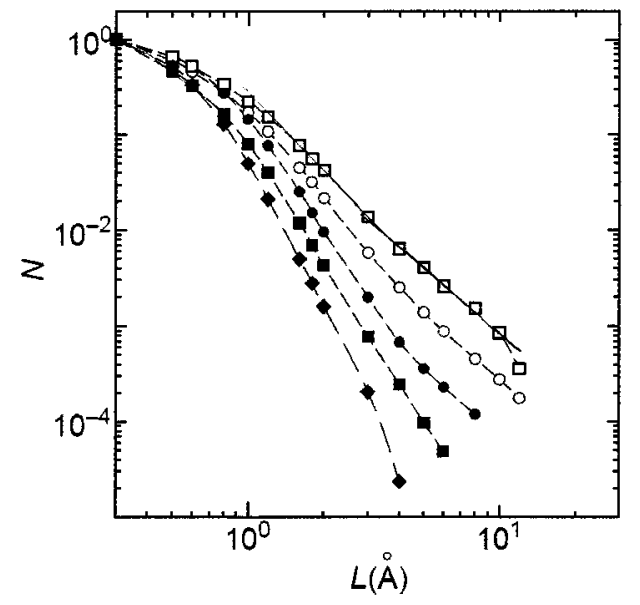

Fig. 3 Temperature dependence of the $N$ against $L$ plot obtained from the trajectories. Fractal dimension of the random walks is obtained from the slope. Open square, open circle, filled circle, filled square and filled diamond are obtained at 600, 400, 370, 350 and 250 $\mathrm{K}$, respectively for cations. The slopes of each short and long $L$ region are different at most temperatures. The fitted lines at $600 \mathrm{~K}$ in each region show inflection at around $L=3$.

dynamical heterogeneity for both temporal terms (waiting time distribution of jumps) and spatial terms (geometrical correlation among successive motions). ${ }^{21}$ The fractal dimension analysis of the trajectories is concerned with the spatial term. The plots of $N$ against $L$ at $600,400,370,350$ and $250 \mathrm{~K}$ for cations are shown in a double logarithmic scale in Fig. 3, where $N$ is normalized for the value at $L=0.3$. From these results, we find the motion has fractal character up to large lengths. The slope in Fig. 3 at $600 \mathrm{~K}$ changes at $3 \AA$ as shown by the fitted lines. Similar inflections are found at about $3 \AA$ at other temperatures except for the lowest temperature. This distance would be typical for that between the nearest anion sites and cation sites. The temperature dependence of the long range motion is stronger than that of the short range motion. In particular, the contribution of the long range motion rapidly decreases with lowering temperature and $N$ of $L>3$ is less than $10^{-3}$ at $350 \mathrm{~K}$ in this plot. At $350 \mathrm{~K}$, the difference of the slopes for the two regions is small. At temperatures higher than this temperature, $d_{\mathrm{w}}$ for longer length scale motion is smaller than that for the shorter length scale motion. On the other hand, an opposite trend is observed at lower temperatures. Long range motion is essentially absent at $250 \mathrm{~K}$. The mean squared displacement in the power law region is proportional to $t^{\theta}$, where $\theta=2 / d_{\mathrm{w}}$, when the temporal term can be neglected. In general, the back correlation probability of ions depends on the fracton dimension defined by $2 d_{\mathrm{f}} / d_{\mathrm{w}}$, ${ }^{22}$ where $d_{\mathrm{f}}$ is the dimension of the path. In the present system, the mobility of the ions is of the same order as that of the surrounding ions; therefore, the changes in the path occur on a time scale similar to that of the motion of the ion itself. Therefore $d_{\mathrm{f}}$ will be comparable to the dimension of the space at high temperature region. Interception of the paths caused by immobile ions at low temperatures will decrease the value, although further study is necessary for a quantitative estimate of this effect. From the slopes of the shorter and longer length scale regions of Fig. 3, $d_{\mathrm{w}}$ for cations was determined as shown in Fig. 4. A rapid increase of $d_{\mathrm{w}}$ with decreasing temperature is observed at around $400 \mathrm{~K}$, and at lower temperatures short length scale motion becomes dominant. A normal (independent) random walk has $d_{\mathrm{w}}=2$, and deviation of $d_{\mathrm{w}}$ from this value for long range motion indicates abnormal 


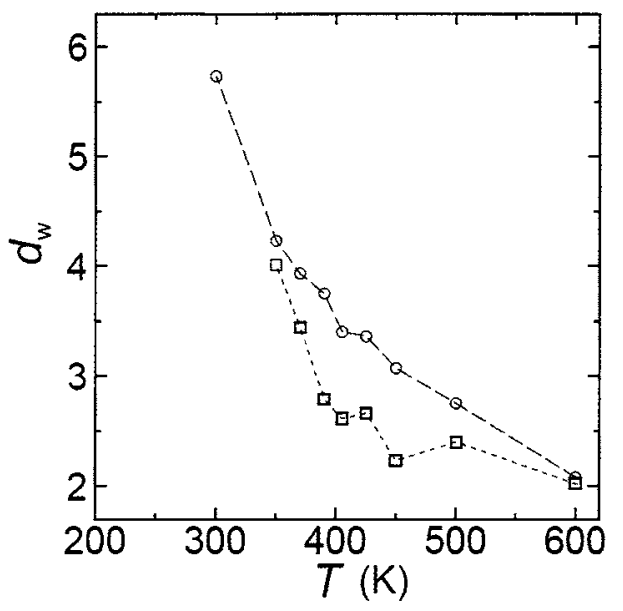

Fig. 4 Temperature dependence of $d_{\mathrm{w}}$ for shorter $L<3$ (circles) and longer length $L>3$ (squares) region for cations. The data are connected to guide the eye.

diffusion. At $600 \mathrm{~K}, d_{\mathrm{w}}=2.08$ for $1<L<3 \AA$ and $d_{\mathrm{w}}=2.02$ for $10>L>3 \AA$. Therefore $d_{\mathrm{w}}$ for long range motion is near the value for the normal random walk (however, note that the value being smaller than that for short range motion means overlap of the fast dynamics, as will be discussed later). With decrease in temperature, $d_{\mathrm{w}}$ gradually increases at the beginning, and a rapid increase is found at around $400 \mathrm{~K}$, where the values of $d_{\mathrm{w}}$ for longer scale motion exceed 3 . For example, at $370 \mathrm{~K}$, we find $d_{\mathrm{w}}=3.93$ for $L<3 \AA$ and $d_{\mathrm{w}}=3.44$ for $L>3 \AA$. Value of $d_{\mathrm{w}}$ larger than 2 is due to the motion having backward correlation, and larger $d_{\mathrm{w}}$ means higher probability of backward correlation. The number 3 for the space dimension is the limit for $d_{\mathrm{w}}$, if overlaps of trajectories are not allowed. The value can be greater than 3 at lower temperatures, because $d_{\mathrm{w}}$ is a latent dimension which includes the folding of the trajectories. Thus the values of $d_{\mathrm{w}}$ larger than 3 mean that the localized trajectories of ions are folded many times and do not easily spread over space at lower temperatures. These changes in $d_{\mathrm{w}}$ values and changes in the contribution of long range motion reasonably explain the inflection observed in the temperature dependence of the diffusion coefficients.

Dynamical heterogeneity was found for many systems ${ }^{23}$ as well as in the ionic liquids, ${ }^{9,24}$ although the definition of the heterogeneity is not necessarily the same in works by different authors. The definition of some researchers is based on the jump rate only; some are concerned with short length scale motion and others are concerned with long range ones. In the present work, "fast and slow" is concerned with the transport properties of long time, rather than short time and short length scale behaviors. The analysis of $d_{\mathrm{w}}$ has been done for all ions, so the slope is an averaged one. However, the dynamical heterogeneity should be taken into account in the explanation of the results. The changes of dynamics are concerned with the suppression of the spreading of the trajectory in the space at around $400 \mathrm{~K}$. Larger values found of $d_{\mathrm{w}}$ at lower temperatures mean the increased backward correlation as well as the decrease in number of long (forward correlated) jumps contributing to the diffusivity. Simultaneous (cooperative) jumps of several atoms were found for binary soft-core systems. ${ }^{25}$ This kind of cooperative jump of several atoms can be followed by either back-correlated motion or by forward-correlated motion. On the other hand, successive jumps of ions were previously known in super ionic conductors ${ }^{26}$ and in glasses. ${ }^{27}$ Jumping ions with forward correlation related with the power law distribution of effective jump length (Lévy flight ${ }^{28}$ ) are found to be the main component contributing diffusive motion at longer time scales ${ }^{29}$ and they reduce the values of $d_{\mathrm{w} 2 .}{ }^{13}$ A relation between cooperative jumps and successive jumps had been pointed out before $^{29}$ and the deterministic character of such motions was clarified later. ${ }^{30}$ Similar motions called "stringlike" motions are also found in other glass forming liquids. ${ }^{31,32}$ Thus dynamics determined by the geometrical character of the trajectories are quite common in ionic and non-ionic systems with or without an immobile network matrix.

When researchers study cooperative motions, the presence of a growing correlation length on approaching the glass transition was frequently assumed from the theoretical framework of conventional critical phenomena. Several efforts to obtain the length scale have been made ${ }^{33}$ but only a small increase with temperatures is found in some length scales (in most cases, it is found near $T_{\mathrm{c}}$ of the mode coupling theory and not near $T_{\mathrm{g}}$ ). However, when the dynamics change rather smoothly, the question is raised whether divergence of the growing length scale is a necessity to explain the glass transition. At least our results in tracer diffusion are not consistent with this proposal of divergence of the length scale, when we consider the relation between single particle motion and collective motion. Conductivity with collective character is known to be related to the tracer diffusivity through the Haven Ratio (typically with a value in the range $0.3-0.5$ in the case of ionic glasses with single alkali, and slightly larger in the mixed alkali systems) as shown in the following relation.

The complex conductivity is a function of the mean squared displacements, ${ }^{34-36}$

$$
\sigma^{*}(\omega)=-\omega^{2} \frac{N_{\text {ion }} q^{2}}{6 H_{\mathrm{R}} k T} \int_{0}^{\infty}<r^{2}(t)>e^{-\mathrm{i} \omega \mathrm{t}} \mathrm{d} t,
$$

where $N_{\text {ion }}$ is the number density of mobile ions, $q$ the ion charge, $k$ the Boltzmann constant, and $T$ the temperature. $H_{\mathrm{R}}$, the Haven ratio, is the ratio of the self diffusion and the bulk diffusion $^{34}$ given by

$$
H_{\mathrm{R}}=\sum_{\mathrm{i}}\left\langle v_{\mathrm{i}}(0) \cdot v_{\mathrm{i}}(t)\right\rangle /\left\langle\sum_{\mathrm{i}} v_{\mathrm{i}}(0) \cdot \sum_{\mathrm{j}} v_{\mathrm{j}}(t)\right\rangle .
$$

Therefore, the trend observed for tracer diffusion should be related to the collective motion, unless a sudden change in the Haven ratio occurs near the glass transition region of ILs. It is worth mentioning that when both anion and cation can move by cooperative jumps in the same direction, this contributes to the diffusion but not the conductivity, and thus this also affects the behavior of the Haven Ratio. In the present IL, the motions of the anion and cation are strongly coupled with each other and their motions are directly related to the structural relaxation, except for a modification by the dynamical heterogeneity. The motions of cation and anion are only slightly decoupled near the glass transition temperature, as shown by the van-Hove function. Therefore, the behaviors of tracer diffusion and collective motion are connected to each other in the present system. When the ionic motion is decoupled from the structural relaxation, the length scale of the cooperative motions for viscosity can be different from that of conductivity or diffusion, as discussed later.

Previously, we have pointed out that the truncation of the Lévy distribution found in ions in glasses is related to the apparent length scale. ${ }^{37}$ At least for mobile ions, the largest length scale observed in the $N$ against $L$ plots is decreasing with lowering temperature rather than increasing ${ }^{38}$ at the low temperature region. The results in the present work are also consistent with 

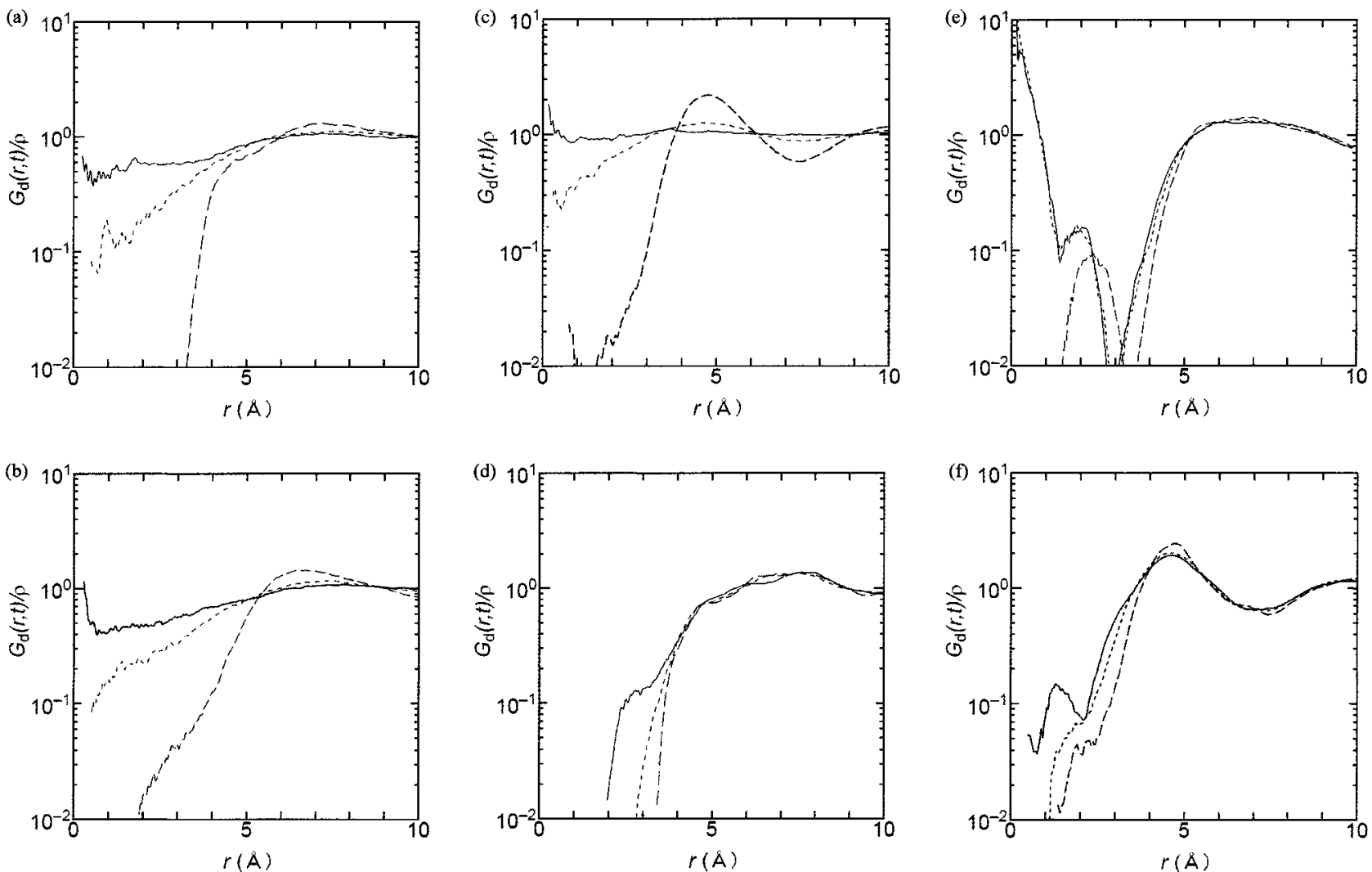

Fig. 5 Distinct parts of the van-Hove functions in the molten state: (a) cation-cation, (b) anion-anion, (c) cationanion pairs at $600 \mathrm{~K}$ at 0 (dashed), 40 (dotted), 80 (solid) ps. Those in the glassy state: (d) cation-cation, (e) anionanion, (f) cation-anion pairs at $250 \mathrm{~K}$ at 0 (dashed), 4 (dotted), 8 (solid) ns. At $600 \mathrm{~K}$, broadening of the original first peak occurs for each pair and the function becomes flatter sooner. The peak at around 0 is found to develop for only anion-anion pair at $250 \mathrm{~K}$. That is, the motion of cations is almost fixed within a cage during this time scale.

this conclusion. That is, the trajectory becomes more compact and local with decreasing temperatures. Due to the long length scale of the motion, contributions of rare events such as the cooperative motion of many ions can not necessarily be neglected for a certain temperature region. However, suppression of long range motion is also observed at lower temperatures in the van-Hove functions, as shown later.

Instead of the divergence of the mobile region, it seems to be more natural to consider the increase of the immobile region with decreasing temperature, because the mobile regions are cut by immobile parts as discussed in the following section.

\section{Dynamics in the Molten and Glassy State}

The distinct part of the van-Hove function is defined by

$$
G_{\mathrm{d}}^{\alpha, \beta}(\vec{r}, t)=\left(1 / N_{\alpha}\right) \sum_{\mathrm{i}=1}^{\mathrm{N}_{\alpha}} \sum_{\mathrm{j}=1}^{\mathrm{N}_{\beta}}\left\langle\delta\left(\vec{r}-\vec{r}_{\mathrm{i}}^{\alpha}(0)+\vec{r}_{\mathrm{j}}^{\beta}(t)\right)\right\rangle,
$$

where in the summations the self-term $i=j$ is to be left out if $\alpha$ $=\beta$. $N_{\alpha}$ and $N_{\beta}$ are the number of particles of species $\alpha$ and $\beta$, respectively. In the function, if the ion species $\beta$ comes into the site previously occupied by species $\alpha$ at an initial time $t_{0}$, a new peak developed at around $r=0$.

In Fig. 5, the functions for the molten state at $600 \mathrm{~K}$ for (a) cation-cation (b) anion-anion and (c) cation-anion pair are shown. The times are at 0,40 and $80 \mathrm{ps}$ in each figure. Those in the glassy state at $250 \mathrm{~K}$ for (d) cation-cation (e) anion-anion and (f) cation-anion pair are also shown. Times are at 0,4 , and
$8 \mathrm{~ns}$ in each figure. The curves for anion-cation pair are approximately the same as those for cation-anion and are not shown here. The function at $t=0$ corresponds to $g(r)$, the pair correlation function. At $600 \mathrm{~K}$, broadening of the original first peak occurs for each pair and the function becomes flatter sooner. The behavior of the cation-anion pair means that the mutual diffusion occurs. On lowering the temperature, the motion becomes jump-like. From the functions obtained at higher temperature, jumps from cation site to anion site (and vice versa) are frequently observed, while they are suppressed at lower temperatures. ${ }^{24}$ At $300 \mathrm{~K}$ (not shown here), only a limited number of local jumps to the next shell are observed during several ns runs, and the mutual diffusion among the sites of anions and cations is rare as is self diffusion.

In the liquid state, the ion in the ionic liquid shows ${ }^{23}$ diffusive jumps as well as large motion in the so-called nearly constant loss (NCL) $)^{39,40}$ region, while in the super-cooled liquid state, the short length scale motions are dominant. That is, the temperature dependence of diffusion coefficients is related to the suppression of the successive jumps with long length scale. At $250 \mathrm{~K}$ shown in Fig. 5(e), new peaks developed at around $r=$ 0 for anion-anion pair with elapse of time ( 4 and $8 \mathrm{~ns}$ ), while the broadening of the original peaks (i.e., at $t=0)$ and development of the small bump are found for other pairs in (d) and (f). Thus the cations are essentially immobile and form immobile domains. In the glassy state, even the jumps of cations among neighboring sites are suppressed, at least during the observation time of this run.

An ionic liquid can be regarded as binary mixture of anions 
and cations. In the binary system, the localization is governed not only by the local structure of the potential function but also by the mutual interception of paths of different kind of species, resulting in the suppression of the cooperative motions at lower temperatures. The situation is similar to that in the mixed alkali glasses ${ }^{41}$ where the mutual interception of jump paths of different kinds of alkali metal ions causes the slowing down of the dynamics. This result is also consistent with the observed changes in $d_{\mathrm{w}}$ values.

MD simulations for the same system had been done previously by Del Popolo and Voth ${ }^{9}$ and the behaviors at $400 \mathrm{~K}$ were examined. Our present results for the pair correlation function of center of mass, $g(r)$ and the diffusion coefficient of both cation and anion at $400 \mathrm{~K}$ are comparable to those obtained by them. The quality of the potential parameters in the present work seems to be comparable to the ones used by them. Pair correlation functions are also comparable with those at a similar system, $\mathrm{DMIM}^{+} \mathrm{Cl}^{-}$(1,3-dimethyl imidazolium chloride), obtained by neutron diffraction experiments. ${ }^{42}$ Several force fields specific to the ionic liquids have been proposed so far, including the polarizable model. ${ }^{43}$ Further study may be necessary to clarify the role of the polarizability in the glass transition problem, although it is not necessarily essential for establishing the general trends observed here.

There are many theories or models to explain the glass transitions and related dynamics. ${ }^{44-49}$ Some of them describe the glass transition as the localization and trapping within a free energy landscape, while others consider it as due to the rapid increase of cooperatively rearranging regions or some length scales near the glass transition temperature. Our results indicate that the long range dynamics are dominant at high temperature regions and are suppressed in the low temperature regimes where more localized dynamics prevail. No diverging length scale for mobile ions with decreasing temperature is found, at least in the present work. The long range dynamics at higher temperature (in the super-cooled liquid state) are related to the accelerated dynamics by cooperative motions. Each jump is not a typical jump and fluctuations of the position of the cage itself contribute to the dynamics at higher temperatures, as will be shown elsewhere. ${ }^{24}$ Therefore, the behavior at higher temperature is more affected by the other ions, and at lower temperature the behavior is governed by the more fixed landscape. We have explained similar behavior of ions in the ion-conducting inorganic glasses as the changes in the density profile (dynamic potential surface) with multifractal characters, ${ }^{50}$ which is combined with Tsallis entropy ${ }^{51}$ and deterministic (cooperative) motion forming the landscape. ${ }^{52}$ Explanations given there will be also applicable for the present system. Our findings seem to be consistent with the description of the glass transition by the energy landscape mode ${ }^{47}$ in some essential parts, although the picture is not necessarily the same. On the other hands, description by the fixed potential energy surface ${ }^{49}$ (especially when $N$ particles are treated as point-like) is not enough to explain our results. The results are also consistent with the changes in the exponents with temperature suggested by the coupling model, since $2 / d_{\mathrm{w}}$ is related to the power law exponent of MSD and corresponding intermediate scattering function $F_{\mathrm{s}}(k, t)$. Further comparison seems to be necessary for discussing the validity of each theory with consideration of some points to be discussed below.

One might expect that the behavior of the diffusivity is connected to the viscosity, $\eta$, through the Stokes-Einstein relation:

$$
D=\frac{k T}{3 \pi \eta d}
$$

where the $d$ is an hydrodynamics diameter of particles placed in the solvent. In MD simulations, the value $\eta$ can be obtained from the off-diagonal elements of the stress tensor or by a nonequilibrium method. If one defines the glass transition by the changes in the viscosity, the situation is slightly changed from the discussions for the diffusivity or conductivity. Different slopes of temperature dependence of diffusivity and viscosity with a collective character resulted in a deviation from the Stokes-Einstein relation as discussed for the ionic liquids,,$^{53}$ molten salts ${ }^{54}$ and in the generalized binary Lennard-Jones systems. ${ }^{55}$ The deviation is expected to be larger when the decoupling of structural and diffusive relaxation is larger. Similar discussions hold for the relations between conductivity and viscosity. Larger deviations are also expected when the heterogeneity of the dynamics is larger. Since the ionic liquid is a binary mixture of cation and anion, the comparison with other binary systems is meaningful. Most of stable glass former is a mixture of several kinds of atoms, where differences in the effective size of the particles and the concept of "coupling and de-coupling" play an important role in determining the dynamics, as confirmed in the mixed alkali systems ${ }^{41}$ as well as the generalized binary Lennard-Jones systems. ${ }^{56}$

\section{Conclusion}

Dynamics near the glass transition regime in ionic liquids exemplified by EMIM- $\mathrm{NO}_{3}$ have been examined by molecular dynamics simulations using an all-atom model. The model shows the glass transition occurs as evidenced by plotting density against temperature. The temperature dependence of diffusion coefficients shows the dynamical slowing down. This can be well fitted to the empirical VFT equation but can be also fitted to exponential functions or power laws in the high and low temperature regions. Fractal dimension analysis of the trajectories and the van-Hove functions show that the changes from longer length scale dynamics to localized dynamics occur along with the changes of the slope in the plot. Therefore, the change is reasonably explained by the different slopes for long range dynamics and local dynamics rather than by a rapid decrease of diffusivity near $T_{0}$ in the VFT function. The dynamics of ions is quite similar to that in other glass forming liquids. Naturally, the conclusions here can be applied to these other systems.

\section{Acknowledgements}

This research was partly supported by the Ministry of Education, Science, Sports and Culture, Japan, Grant-in-Aid for Scientific Research (C), 19540396, 2007-2009. The work performed at the Naval Research Laboratory was supported by the Office of Naval Research.

\section{References}

1. T. Welton, Chem. Rev., 1999, 99, 2071.

2. M. J. Earle and K. R. Seddon, Pure Appl. Chem., 2000, 72, 1398.

3. P. Wasserscheid and W. Keim, Angew. Chem., Int. Ed., 2000, 39, 3772 .

4. T. Kitazume, J. Fluorine Chem., 2000, 105, 265.

5. T. I. Morrow and E. J. Maginn, J. Phys. Chem. B, 2002, 106, 12807. 
6. J. N. C. Lopes, J. Deschamps, and A. A. H. Pádua, J. Phys. Chem. B, 2004, 108, 2038.

7. C. G. Hanke, S. L. Price, and R. M. Lynden-Bell, Mol. Phys., 2001, 99, 801.

8. C. J. Margulis, Mol. Phys., 2004, 102, 829.

9. M. G. Del Popolo and G. A. Voth, J. Phys. Chem. B, 2004, 108, 1744.

10. X. P. Wu, Z. P. Liu, S. P. Huang, and W. C. Wang, Phys. Chem. Chem. Phys., 2005, 7, 2771.

11. J. Wang, R. M. Wolf, J. W. Caldwell, P. A. Kallamn, and D. A. Case, J. Comput. Chem., 2004, 25, 1157.

12. B. Mandelbrot, Science, 1967, 155, 636.

13. J. Habasaki and K. L. Ngai, J. Chem. Phys., 2004, 120, 8195.

14. H. Cang, J. Li, and M. D. Fayer, J. Chem. Phys., 2003, 119, 13017.

15. O. Yamamuro, Y. Minamimoto, Y. Inamura, S. Hayashi, and H. Hamaguchi, Chem. Phys. Lett., 2006, 423, 371.

16. P. Bordat, F. Affouard, M. Descamps, and K. L. Ngai, Phys. Rev. Lett., 2004, 93, 105502 and references herein.

17. P. Bordat, F. Affouard, M. Descamps, and K. L. Ngai, J. Non-Cryst. Solids, 2006, 352, 4630.

18. H. Tokuda, K. Hayamizu, K. Ishii, A. B. H. Susan, and M. Watanabe, J. Phys. Chem. B, 2004, 108(42), 16593.

19. J. Vila, P. Ginés, J. M. Pico, C. Franjo, E. Jiménez, L. M. Varela, and O. Cabeza, Fluid Phase Equilib., 2006, 242, 141.

20. H. Vogel, Phys. Zeit., 1921, 22, 645; G. S. Fulcher, J. Am. Ceram. Soc., 1925, 8, 339.

21. J. Habasaki, I. Okada, and Y. Hiwatari, Phys. Rev. E, 1995, 52, 2681; 1998, 67, 2012.

22. S. Alexander and R. Orbach, J. Phys. (Paris) Lett., 1982, 43, L625.

23. H. Sillescu, J. Non-Cryst. Solids, 1999, 243, 81.

24. J. Habasaki and K. Ngai, submitted to J. Chem. Phys.

25. H. Miyagawa, Y. Hiwatari, B. Bernu, and J. P. Hansen, J. Chem. Phys., 1988, 88, 3879.

26. Y. Kaneko and A. Ueda, "Molecular Dynamics Simulations", ed. F. Yonezawa, 1992, Vol. 103, Springer, New York, 248.

27. J. Habasaki, I. Okada, and Y. Hiwatari, J. Non-Cryst. Solids, 1996, 208, 181.

28. M. F. Shlesinger, G. M. Zaslavsky, and J. Klafter, Nature [London, U. K.], 1993, 363, 31; J. Klafter, M. F. Shlesinger, and G. Zumofen, Phys. Today, 1996, 49(2), 33.

29. J. Habasaki, I. Okada, and Y. Hiwatari, Phys. Rev. B, 1997, 55,6309 .
30. J. Habasaki, K. L. Ngai, and Y. Hiwatari, J. Chem. Phys., 2004, 120, 8175; J. Habasaki, J. Non-Cryst. Solids, 2007, $353,3956$.

31. Y. Hiwatari, J. Habasaki, and T. Muranaka, 3rd International Discussion on Relaxations in Complex Systems, 1997, Vigo.

32. C. Donati, J. F. Douglas, W. Kob, S. J. Plimton, P. H. Poole, and S. C. Glotzer, Phys. Rev. Lett., 1998, 80, 2338.

33. N. Lacevic, F. W. Starr, T. B. Schroder, and S. C. Glotzer, J. Chem. Phys., 2003, 119, 7372 and references herein.

34. R. Kubo, J. Phys. Soc. Jpn., 1957, 12, 570.

35. T. Odagaki and M. Lax, Phys. Rev. B, 1981, 24, 5284.

36. K. Funke and R. D. Banhatti, J. Non-Cryst. Solids, 2007, $353,3845$.

37. J. Habasaki and K. L. Ngai, J. Chem. Phys., 2005, 122, 214725 .

38. J. Habasaki and K. L. Ngai, J. Chem. Phys., 2008, 129, 034503.

39. K. L. Ngai, J. Habasaki, Y. Hiwatari, and C. Leon, J. Phys.: Condens. Matter, 2003, 15, S1606.

40. K. L. Ngai and C. Leon, Phys. Rev. B, 2002, 66, 064308.

41. J. Habasaki and K. L. Ngai, Phys. Chem. Chem. Phys., 2007, 9, 4662 and references herein.

42. C. Hardacre, J. D. Holbrey, S. E. J. McMath, D. T. Bowron, and A. Soper, J. Chem. Phys., 2003, 118, 273.

43. T. Yan, C. J. Burnam, M. G. Del Popolo, and G. V. Voth, J. Phys. Chem. B, 2004, 108, 11877.

44. G. Adams and J. H. Gibbs, J. Chem. Phys., 1965, 43, 139.

45. E. Leutheusser, Phys. Rev. A, 1984, 29, 2765.

46. T. Odagaki and Y. Hiwatari, Phys. Rev. A, 1990, 41, 929.

47. T. Odagaki and T. Ekimoto, J. Non-Cryst. Solids, 2007, 353, 2928.

48. K. L. Ngai, R. W. Rendell, and D. J. Plazek, J. Chem. Phys., 1991, 94, 3018.

49. F. Sciortino, J. Stat. Mech., 2005, 05, 5015.

50. J. Habasaki and K. L. Ngai, J. Chem. Phys., 2005, 122, 214725.

51. M. L. Lyra and C. Tsallis, Phys. Rev. Lett., 1998, 80, 53.

52. J. Habasaki, K. L. Ngai, and Y. Hiwatari, J. Chem. Phys., 2005, 122, 054507.

53. K. L. Ngai, J. Phys. Chem. B, 2006, 110, 26211.

54. P. Singh, R. D. Banhatti, and K. Funke, Phys. Chem. Chem. Phys., 2005, 7, 1096.

55. J. Habasaki, F. Affouard, M. Descamps, and K. L. Ngai, in Proceedings of AIP Conference 982, 2008, 154.

56. J. Habasaki, F. Affouard, M. Descamps, and K. L. Ngai, unpublished. 